\title{
Anxiety: a hidden element in dementia
}

\author{
Vellingiri Raja Badrakalimuthu \& Andrew F. Tarbuck
}

\begin{abstract}
SUMMARY
Anxiety has reported prevalence rates between 38 and $72 \%$ among people with dementia. It has a negative impact on cognitive impairment and is associated with agitation and poor quality of life. The presence of excessive anxiety can be difficult to establish in people with dementia, especially when expressive or receptive speech is impaired. Unfortunately, there is a lack of research on the treatment of anxiety in dementia, and also on the wider issue of the management of anxiety disorders in old age. We explore the prevalence, presentation and diagnosis of anxiety in dementia and discuss the therapeutic options available.
\end{abstract}

\section{DECLARATION OF INTEREST}

None.

Generalised anxiety disorder is the most common late-life psychiatric disorder. It has a community prevalence of $2-7 \%$, and a prevalence of around $10 \%$ in patients presenting to primary care (Beekman 1998). In people with dementia, however, the rate rises to between 38 and $72 \%$ (Ballard 2000).

Many studies have looked at factors influencing the presentation of anxiety in dementia. No relationship has been found with age, gender (Ballard 2000) or education (Mendez 2006). In the USA, higher rates of anxiety in dementia have been found among Hispanics and Asians than White or African Americans (Chen 2000). Anxiety is more common in vascular (Ballard 2000), frontotemporal and Parkinson's dementias (Aarsland 2001) than in other dementia syndromes. Anxiety in dementia is associated with retention of insight and awareness (Seignourel 2008), and there is evidence to suggest that levels of anxiety are lower in profound/severe dementia (Chen 2000). Anxiety in dementia is associated with poor quality of life (Banerjee 2006) and unmet needs among residents in care homes (Hancock 2006).

\section{Impact on cognitive decline across the course of dementia}

Behavioural and psychological symptoms of dementia are not limited to the later stages of the disease. A study of anxiety in elderly people with mild cognitive impairment (Rozzini 2009) reported that individuals with a Generalised Anxiety Inventory score $\geq 10$ showed more behavioural and psychological disturbances, agitation, anxiety, depression and sleep disorders. They were also more compromised on instrumental activities of daily living and on executive functions evaluated using the Trail Making B test. High levels of anxiety in patients with mild cognitive impairment have an adverse effect on executive functioning.

In early Alzheimer's disease, anxiety (but not depression) is reported to predict a decline in learning as measured on the Auditory Verbal Learning Test (Bierman 2009).

In a prospective study of anxiety and cognition in 1481 men aged between 48 and 67, participants were reassessed after 17 years' follow-up (Gallacher 2009). Vascular risk factors and poorer premorbid cognitive function were significantly associated with anxiety, and decline in learning and memory was associated with high baseline anxiety scores.

Older adults reporting high levels of anxiety and depression perform poorly on tasks assessing attention and concentration, set-shifting, and working and episodic memory (Derousene 2004). The relationship between anxiety and cognitive performance is complex, probably because of non-linear interactions between anxiety and test difficulty.

\section{Agitation}

Agitation is one of the most common and severe of the neuropsychiatric symptoms of dementia. It has been variously estimated that almost 60\% (Mintzer 1996) and over 70\% (Cohen-Mansfield 1986) of people with dementia present with agitation during the course of their illness.

Mintzer \& Brawman-Mintzer (1996), in a review of generalised anxiety disorder in dementia, suggest that agitation is commonly due to underlying anxiety. Twelftree \& Qazi (2006) also report a link between anxiety and agitation in dementia. However, Seignourel et al (2008) state that the evidence base favours treating anxiety and agitation as distinct entities, rather than considering them to be closely linked. Another difficulty in determining associations is that a reported $75 \%$ of those who meet criteria for dementia plus generalised anxiety disorder also fulfil diagnostic criteria for depression (Starkstein 2007).

\section{ARTICLE}

Vellingiri Raja Badrakalimuthu is a specialty registrar in old age psychiatry at Fulbourn Hospital, Cambridge. His research interests include psychopharmacology and dementia. Andrew Tarbuck is a consultant in old age psychiatry with Norfolk and Waveney Mental Health NHS Foundation Trust, working at the Julian Hospital, Norwich, and an honorary senior lecturer at the University of East Anglia. He is also Director of the DeNDRoN East Anglia Research Network. He is interested in medical education and promoting research into neurodegenerative disorders. Correspondence Dr V. R. Badrakalimuthu, Beechcroft, Fulbourn Hospital, Cambridge CB21 5EF, UK. Email: raja.badrakalimuthu@cpft. nhs.uk 
BOX 1 Physical disorders that can confound the diagnosis of anxiety in dementia

- Thyroid dysfunction

- Hypoglycaemia

- Angina

- Congestive cardiac failure

- Chronic obstructive pulmonary disease

- Tumour

- Parkinson's disease and other neurodegenerative disorders

\section{Diagnosing anxiety in dementia}

Making the diagnosis of anxiety in someone with dementia requires a comprehensive assessment. This is necessary not only to confirm the presence of anxiety symptoms, but also to explore possible biopsychosocial aetiological factors involved.

Physical examination and laboratory investigations are important for the identification and appropriate treatment of physical disorders that predispose to, precipitate or mimic anxiety (Box 1). Prescribed medications such as steroids, antidepressants and anticholinergics can cause anxiety, and alcohol use should always be assessed.

The presence of anxiety can be difficult to establish in people with dementia, especially when expressive or receptive language is impaired. Neurodegenerative changes associated with dementia can involve limbic areas such as the hippocampus and amygdala, which are implicated in the regulation of emotions, including anxiety. Starkstein et al (2007) report a number of symptoms that can individually predict the presence of generalised anxiety disorder in dementia: excessive anxiety or worry that is difficult to control, restlessness, irritability, muscle tension, fears and respiratory distress.

It is important to look for evidence of depression, as it is often associated with anxiety and agitation. A simple 'ABC' (antecedents, behaviours, consequences) functional analysis of behavioural problems may be helpful, as it may reveal the presence of additional psychosocial factors related to anxiety.

\section{Rating scales}

There are few structured and standardised measures for screening, diagnosing and rating the severity of anxiety comorbid with dementia (Box 2), and further research is required to address this paucity.

One dementia-specific instrument is the Rating of Anxiety In Dementia (RAID) (Shankar 1999). This 20-item scale has good interrater reliability, sensitivity and specificity. It correlates highly with the DSM-IV diagnosis of generalised anxiety disorder (American Psychiatric Association 1994), but it has been used infrequently in research.

High correlations have been reported between the anxiety domains of the Behavioral Pathology in Alzheimer's Disease (BEHAVE-AD) rating scale and the Neuropsychiatric Inventory (Cummings 1994).

The Worry Scale (LaBarge 1993), which is designed as a self-report measure for anxiety in mild dementia, has good internal consistency and correlation with trait and state anxiety, and it also correlates strongly with measures of depression. Other scales that are also sensitive to the presence of depression include the Hospital Anxiety and Depression Scale (HADS), although this was not designed for use in dementia. A review of the HADS in 747 studies involving elderly people (but not with a diagnosis of dementia) reports that it has adequate sensitivity and specificity as a screening tool of both anxiety and depression (Bjelland 2002). Thus, it might be used as a screening tool in elderly patients with mild to moderate dementia who can still communicate.

The Schedule for Clinical Assessment in Neuropsychiatry (SCAN; Wing 1990) includes sections focusing on anxiety disorders and mood disorders. The section on anxiety, for example, includes direct questions to establish the degree of worrying and tension felt by the patient. The sections also include observation items, making SCAN useful in the bedside evaluation of patients with dementia who have limited communication.

\section{Psychological treatments}

A survey by Evans (2004) found that 95\% of UK mental health services for older adults offered some form of psychological treatment for anxiety disorder, despite generally inadequate training

BOX 2 Bedside tools for assessment of anxiety in dementia

- Worry Scale (LaBarge 1993): self-reported measure of anxiety in mild dementia

- Rating of Anxiety In Dementia (RAID) (Shankar 1999): in severe dementia where communication is restricted and care-givers' reports have to be taken into account

- Hospital Anxiety and Depression Scale (HADS) (Bjelland 2002): screening tool for anxiety in mild to moderate dementia where communication is possible

- Neuropsychiatric Inventory (Cummings 1994): can be used in conjunction with the RAID to explore interaction between anxiety and behavioural and psychological symptoms of dementia 
and supervision of staff delivering the therapy. Behavioural therapy, individual and group cognitive-behavioural therapy (CBT), interpersonal therapy, reality orientation, validation therapy and reminiscence therapy have all been associated with some success in managing various symptoms of dementia. However, there has been a lack of studies looking specifically at the management of anxiety in people with dementia.

Box 3 summarises dementia-specific psychological treatments that have been shown to be efficacious in managing agitation and improving mood. Since anxiety is a common factor associated with depression and agitation, these interventions may be useful in managing anxiety in dementia.

\section{Anxiety-focused treatments}

Counselling

An increased awareness of dementia among the general public, coupled with media reports of the advent of specific drug treatments for dementia (the cholinesterase inhibitors donepezil, rivastigmine and galantamine), has led to patients presenting earlier in the illness. Furthermore, they are often more aware of the diagnostic process and its implications for them and their family. Patients presenting in the early stages of dementia can pose significant diagnostic difficulties. Often, they will need to undergo neuropsychological testing and

BOX 3 Summary of evidence for various psychological treatments in dementia

- Reminiscence therapy (Woods 2005) uses materials such as old newspapers and household items to stimulate memories and enable people to share and value their experiences. At least one study reports benefits in terms of better mood.

- Validation therapy (Neal 2003) is intended to give an opportunity to resolve unfinished conflicts by encouraging and validating expression of feelings. The therapy does not appear to reduce nursing time or use of psychotropic medication and restraint.

- Reality orientation therapy (Spector 2000) is based on the idea that impairment in orienting information prevents people with dementia from functioning well and that reminders can improve functioning. There are studies that report improved mood, reduction of neuropsychiatric symptoms and reduced need for psychotropic medication.

- Cognitive stimulation therapy (Spector 2003) uses information processing rather than factual knowledge to address problems in functioning in people with dementia. Although there has been report of improvement of depression, some studies suggest an absence of long-term benefit.
BOX 4 Stages of genetic counselling
1 Confirm the diagnosis
2 Take a comprehensive family history
3 Establish risk in the family
4 Assess mental state and provide a psychosocial formulation
5 Establish the risk/burden ratio through detailed exploration and negotiation
6 Form a plan of action, which will include coping with the illness and coping with the impact on social functioning
7 Follow-up
(Tsuang 1978)

neuroimaging. These processes can be anxietyprovoking in themselves, as can be the delays involved in waiting for tests and for feedback of test results. At the end of the process, a clear diagnosis cannot always be given, or patients are told that they have 'mild cognitive impairment', with its implications of uncertainty and elevated risk of the subsequent development of dementia.

To reduce the risk that significant anxiety will develop during or as a result of the diagnostic process itself, it is important to ensure that an appropriate level of pre- and post-diagnostic counselling and support is available for both patients and their families.

In some cases there may be a clear genetic component to the presentation. The knowledge of genetic information about an individual's risk of dementia due to familial illnesses such as Alzheimer's disease or Huntington's disease raises many ethical, moral, social and legal issues. These in themselves can be triggers for anxiety and it is pertinent that testing is accompanied by rigorous genetic counselling (Box 4) (Tsuang 1978).

If counselling does not lessen anxiety, it is essential that the psychological formulation be reviewed and that other psychological and pharmacological treatments be pursued as appropriate.

\section{Cognitive-behavioural therapy}

Studies on CBT in older adults have focused largely on treating generalised anxiety disorder, panic disorder, subjective worry and/or agoraphobia. From their 15 studies of CBT for elderly people with anxiety, Nordhus \& Pallesan (2003) report an effect size of 0.55 , which is highly significant. This indicates that CBT may be an option for older adults with anxiety comorbid with mild dementia or in the immediate aftermath of a diagnosis of dementia. Group-based CBT and tapering of pharmacological treatment concomitant with CBT may dilute the 
BOX 5 Issues regarding CBT trials for anxiety disorder in elderly people

- Difficulty in setting up a meaningful control group in clinical trials involving CBT

- Most studies exclude participants with cognitive impairment

- Homogeneity and relatively young age of the samples in most trials

- Lack of long-term follow-up

- Difficulty in identifying the essential ingredient of treatment, as some trials involve both group and oneto-one therapy

effects of the therapy. In their studies of CBT for late-life generalised anxiety disorder, Mohlman et al (2003) report that success is dependent on the absence of executive dysfunction at the start of therapy or executive dysfunction that improves during CBT. They also suggest using learning and memory aids, delivery in the patient's own home and cognitive rehabilitation to overcome the barriers in treatment. The person-centred technique of cognitive rehabilitation involves recognising and meeting the individual's needs and goals either by using personalised strategies for registering new information or by using memory aids. An alternative approach, according to Gorenstein \& Papp (2007), is to add supportive group therapy alongside individual CBT.

It should be noted that the evidence base for CBT with older adults is confounded by study limitations. Some of these are specific to the delivery of the CBT, and others arise from the nature of the study population (Box 5).

The components of CBT suitable for elderly people (Box 6) may not be appropriate for individuals with dementia. However, CBT programmes can be simplified: using basic principles such as distraction and relaxation may be sufficient, rather than focusing on in-depth therapy, which may be impossible because of difficulties in communication and cognition.

\section{Problem-solving therapy}

Problem-solving therapy (Box 7) is beneficial in treating depression in elderly people with impaired executive function (Alexopoulos 2011). The premise of the research is that the depression is a symptom in part caused by difficulty in coping with psychosocial problems.

Problem-solving therapy has been used successfully to treat many psychological problems in the general population. The work by Alexopoulos
BOX 6 Components of CBT for anxiety disorders in elderly people

- Education about anxiety symptoms and triggers

- Education about monitoring symptoms

- Progressive, passive and cue-controlled training

- Cognitive restructuring, involving the challenging of thoughts

- Systematic desensitisation

- Imaginary and real-life exposure to anxiety cues and triggers

and colleagues on depression in late-life executive dysfunction raises the hope that a similar intervention may be suitable for patients with moderate to severe dementia presenting with anxiety.

In summary, there is evidence that behavioural interventions and CBT are beneficial for anxiety or anxiety associated with agitation. However, further research is required in identifying specific psychosocial interventions targeting anxiety in people with dementia.

\section{Social interventions}

Anxiety can be triggered or exacerbated by many factors. An older person may be worried that they will lose their balance and fall, or be concerned about their health, financial matters or housing problems; they may feel anxious about future loneliness and social isolation. High levels of anxiety have negative social outcomes, such as reluctance or refusal to leave the house or increased requests for help or attention. These must be fully assessed and care plans should identify appropriate measures to deal with them. Involving occupational therapists, physiotherapists and social workers will be important in developing and implementing an appropriate and adequate social care package. In addition to practical assistance such as home

B0X 7 Stages of problem-solving therapy

1 Selecting and defining problems

2 Establishing realistic goals

3 Generating alternative solutions to meet realistic goals

4 Implementing decision-making guidelines

5 Evaluating and choosing solutions

6 Implementing the preferred solution

7 Evaluating the outcome

8 Activity scheduling

(Modified from Mynors-Wallis 2001) 
BOX 8 Examples of assistive technology for dementia

Memory aids

- Diary, notebook or notice board for reminders of appointments, important phone numbers, etc.

- Automatic calendar clocks

- Locator devices to attach to frequently lost items

- Labels on cupboards or rooms to help remember where things are

- Aids for reminiscence and leisure (e.g. multimedia software for showing family photographs or videos)

- Reminder messages, e.g. to provide reassurance, such as 'Go back to bed, Mum - it's night time'

Telecare

- Sensors, e.g. to detect a flooded floor or scalding bath

- Personal sensor worn on the body to detect falls

(Alzheimer's Society 2011)

care and ensuring that appropriate social security benefits are claimed, increased social contact and stimulation through day care and other social activities can be very helpful. The provision of occupational therapy aids and assistive technology (Box 8) can also be beneficial. Such measures can improve safety in a familiar environment and can be reassuring and reduce anxiety.

Of all groups of carers, those providing care for people with dementia are among the most stressed, and carer stress resulting from behavioural and psychological symptoms of dementia is associated with change of place of care to a care home (Cohen 1990; Shah 2010). Caring for people with dementia is anxiety-provoking and therefore regular assessments of carers' needs and of the support available to them are essential. The carer's burden of care and distress can exacerbate anxiety in the person for whom they care. In dementia care, the dynamic interplay of the carer's and the patient's anxiety may destabilise an already fragile social situation. Carers can be very useful in analysing the precipitants of anxiety in people with dementia and their input will be crucial in offering person-centred care tailored to reducing patients' anxiety.

\section{Pharmacological treatment}

There is a paucity of research exploring drug treatment of anxiety in older people, in particular those with dementia and anxiety. Consequently, treatment strategies have to be largely extrapolated from studies conducted in younger groups of patients who have anxiety but not dementia. The British Association for Psychopharmacology guidelines on treatment of anxiety state that most people who fulfil the criteria for anxiety disorder do benefit from pharmacological treatment (Baldwin 2005). They recommend that the decision to prescribe medication should be based on the severity and duration of symptoms, comorbid conditions, concurrent medications and response to treatments in the past. These guidelines draw on the National Institute for Health and Clinical Excellence (NICE) recommendations regarding the provision of psychological treatments by suitably trained staff (National Collaborating Centre for Mental Health 2011), but comment on the current lack of such individuals. This is a significant problem in dementia care, exacerbated by the paucity of evidence-based psychosocial interventions for anxiety in this specific population and the consequent failure of service provision.

Needless to say, prescribing psychotropic drugs to older people must be undertaken with particular care, and Boxes 9 and 10 offer some reminders on the subject.

\section{Antidepressants}

As discussed earlier, depression can present with anxiety. A meta-analysis of five studies (Thompson 2007) examining the treatment of depression in people with dementia using the tricyclic antidepressants imipramine and clomipramine, and the selective serotonin reuptake inhibitors (SSRIs) sertraline and fluoxetine, showed an overall treatment response and remission rate superior to placebo. However, a subgroup analysis demonstrates that the tricyclics are associated with a significant decline in cognitive scores.

\section{BOX 9 General guidelines in prescribing psychotropics to older people}

- Start low and go slow

- Anticipate and proactively monitor adverse effects of drug interactions

- Encourage patient adherence

- Communicate regularly with the patient and their general practitioner

- Define acceptable measures of response and focus on functional outcomes acceptable to the patient

- Include psychosocial interventions

- Educate the patient on the illness and its treatment

- Discontinue medications that do not yield the expected response

- Change only one medication at a time

- Use the simplest medication regimen 
BOX 10 Pharmacokinetics in old age

\begin{tabular}{|c|c|}
\hline Absorption & Metabolism \\
\hline Gut motility and acid secretion are reduced & $\begin{array}{l}\text { No change in pharmacokinetics unless there } \\
\text { is sianificant liver disease }\end{array}$ \\
\hline $\begin{array}{l}\text { Consequence: slower, but not less, } \\
\text { absorption }\end{array}$ & mulation of drugs in \\
\hline Distribution & \\
\hline Typically, more body fat, less water and & Excretion \\
\hline albumin & $\begin{array}{l}\text { Reduced to two-thirds of normal function in } \\
\text { adults of working age }\end{array}$ \\
\hline for fat-soluble drugs & Consequence: drugs primarily excreted by \\
\hline nsequence: increased amount of & the kidneys will accumulate, causing toxicity \\
\hline
\end{tabular}

The American Psychiatric Association recommends SSRIs as first-line medication for depression in dementia (Rabins 2007). The NICE guidelines (National Collaborating Centre for Mental Health 2006) recommend that depression and/or anxiety in dementia be treated in accordance with the (now updated) guidelines on depression (National Collaborating Centre for Mental Health 2009). In particular, antidepressants with anticholinergic effects, such as tricyclics, should be avoided. The Scottish Intercollegiate Guidelines Network (SIGN) suggests that there is a lack of evidence in support of any particular antidepressant in dementia. Only sertraline has some evidence in its favour, although the sample size was small (Scottish Intercollegiate Guidelines Network 2006).

A review of pharmacological treatments for neuropsychiatric symptoms in dementia reported that, of the antidepressants, citalopram was the only one to be of benefit. It produced a 10-point reduction in the Neurobehavioural Rating Scale, with agitation and lability showing significant improvement compared with placebo (Sink 2005).

Katz et al (2002) have published a pooled analysis of five randomised controlled trials in which venlafaxine was given for generalised anxiety disorder in adults $(n=1839)$. In the subpopulation of patients aged over 60 (10\% of the original sample), venlafaxine was superior to placebo, with benefits seen at 8 and 24 weeks and with an adverse effect profile no different from that of placebo. However, this study did not take into account the degree of cognitive impairment in the study population.

Mirtazapine has also been studied in adults as a treatment for generalised anxiety disorder. In one small study of 44 participants (Gambi 2005), mirtazapine was associated with a $79 \%$ response (defined as a 50\% reduction on the Hamilton Rating Scale for Anxiety, HRSA) and 36\% remission (HRSA score $<7$ ). Thus, mirtazapine may be an option for anxiety, although there are no specific trials in the older population, let alone in a population with dementia.

Trazodone lacks specific trials exploring its use in managing anxiety in either elderly people or people with dementia. Some studies have reported that the drug reduces agitation associated with dementia, and a Cochrane review has concluded that there may be evidence for small improvement in agitation associated with frontotemporal but not Alzheimer's dementia (Martinón-Torres 2004).

A summary of the evidence on antidepressants discussed here is presented in Box 11.

\section{Anxiolytics}

Benzodiazepines are associated with tolerance, dependence and a withdrawal syndrome that can exacerbate cognitive impairment or behavioural disorder. Benzodiazepines with a short halflife (e.g. lorazepam) are eliminated much more quickly than those with a long half-life (e.g. diazepam or alprazolam) and therefore may cause fewer problems of accumulation and consequent toxicity and 'hangover' effects. However, shortacting benzodiazepines are more likely to produce withdrawal symptoms and have a greater propensity to induce dependence.

There are some circumstances (e.g. the use of clonazepam in dementia with Lewy bodies) in which benzodiazepines may be a first-line choice for the management of anxiety and associated behavioural problems. The British Association for Psychopharmacology guidelines (Baldwin 2005) report evidence in support of the use of diazepam and alprazolam in acute anxiety, but warn about the adverse effects.

B0X 11 Evidence on antidepressants in the treatment of anxiety in dementia

\section{Tricyclics}

- Imipramine and clomipramine - evidence of efficacy, but associated with decline in cognitive scores

SSRIs

- Citalopram - evidence of efficacy and associated with reduction in behavioural symptoms of dementia

- Sertraline - evidence of efficacy for anxiety, but no improvement in behavioural symptoms

- Fluoxetine - evidence of efficacy

Others

- Venlafaxine - evidence of efficacy for anxiety in elderly people, but not specifically in people with dementia

- Mirtazapine - evidence of efficacy for anxiety in working-age adults, but no studies in elderly people or in people with dementia 
The advantage of using diazepam and oxazepam in older adults is less risk of dependence but, like all benzodiazepines, they do have particular adverse effects in this population. They may contribute to respiratory insufficiency in patients with pulmonary disorders, and they potentiate the effects of alcohol and opioids. Benzodiazepine use in older people is associated with falls (Neutal 1996), hip fracture (Wagner 2004) and cognitive impairment (Foy 1997). Benzodiazepine withdrawal delirium occurs most frequently in older people, giving rise to an increased rate of neuropsychiatric complications (Wetterling 2002). Distressing discontinuation symptoms occur in as many as $90 \%$ of older patients who have been taking low daily doses. Symptoms such as irritability or restlessness are common. Psychosis, seizures and delirium, although uncommon, can last for 2-4 weeks.

Benzodiazepines with shorter half-life and the so-called Z-drugs (zopiclone, zolpidem, zaleplon), which act on the $\gamma$-aminobutyric acid (GABA) receptors, are efficacious treatments for insomnia (Wilson 2010). However, the interplay between pharmacokinetics and age should be taken into account before prescribing Z-drugs for insomnia that is due to anxiety.

The prescription of benzodiazepines to older people with dementia has to be decided on an individual basis, taking into account comorbid physical illnesses, concurrent medications, the risks of treating $v$. not treating, potential benefits of treatment and whether other psychosocial interventions could be successfully provided. Benzodiazepines have to be used with caution, at the lowest effective dose for the shortest period of time.

\section{Cholinesterase inhibitors and memantine}

No published trials have specifically studied the use of acetylcholinesterase inhibitors to manage anxiety in dementia. There have been secondary analyses of specific symptoms on the Neuropsychiatric Inventory responding to cholinesterase inhibitors. Acetylcholinesterase inhibitors have been shown to be effective in treating depression, dysphoria, anxiety and apathy. They may also reduce aggression and agitation (Gauthier 2002). There is no evidence supporting memantine for the treatment of anxiety in dementia.

Nevertheless, these medications do show benefit in treating cognitive symptoms of dementia and they are safer than antipsychotics (National Collaborating Centre for Mental Health 2006). A rational approach might be to try them after or together with psychosocial interventions if behavioural disturbances are secondary to anxiety or anxiety is the predominant neuropsychiatric symptom.

\section{Antipsychotics}

The most significant adverse consequences of antipsychotic treatment in people with dementia are accelerated cognitive decline and death from cerebrovascular effects.

In a 3-month, open-labelled study of risperidone involving 15 elderly patients with anxiety disorder who had failed to show a therapeutic response to antidepressant and benzodiazepine treatment, Morinigo et al (2005) report of a response in 13 patients. Quetiapine has been effective as an addon to SSRIs or venlafaxine in a study of anxiety comorbid with depression in 58 adults (McIntyre 2006). However, caution should be exercised in extrapolating these findings to the management of anxiety in dementia, because of the risk of adverse effects (including sedation and cerebrovascular events) in older people.

In summary, antipsychotics should be used only in very special circumstances for the management of anxiety in dementia (e.g. severe, intractable anxiety that results in great distress to the patient or produces severe behavioural disturbance).

\section{Anticonvulsants}

Kinrys \&Wygant's (2005) review of anticonvulsants in anxiety disorders, though not specifically related to old age or dementia, suggests that tiagabine, levitiracetam, sodium valproate and carbamazepine have a potential role in the treatment of different forms of anxiety disorder as monotherapy or to augment traditional antidepressants or benzodiazepines. However, the decision to prescribe any of the anticonvulsants to elderly patients with dementia has to be balanced against the adverse effects of these medications.

The common side-effects of anticonvulsants include sedation and cognitive impairment. Less common side-effects include weight gain, metabolic syndrome, impaired liver function and haematological toxicity. Serious adverse consequences include liver and bone-marrow toxicity. In light of the seriousness of the adverse effects, haematological indices, liver function, renal function and weight must be monitored regularly.

\section{Pregabalin}

In a trial of pregabalin for generalised anxiety disorder in 273 elderly patients, the drug produced a 2-point greater reduction than placebo in HRSA total score, evident by the second week of treatment (Montgomery 2008). It was well tolerated, with a discontinuation rate similar to that for placebo. Dizziness, somnolence, headache, nausea and infection were the most common adverse events. Although there is lack of direct evidence for use of 
pregabalin in people with dementia, if patients do not respond to antidepressants and benzodiazepines, pregabalin may be a safer alternative than anticonvulsants and antipsychotics.

\section{Buspirone}

Buspirone is safer than the benzodiazepines as it is not associated with sedation, psychomotor impairment or dependence. In a study involving 40 elderly patients with anxiety in primary care, buspirone was effective (as measured by the HRSA and Clinical Global Impression scale) and well tolerated, with an adverse effect profile similar to that of placebo (Bohm 1990). Again, however, there is no direct evidence to support its use for anxiety in dementia.

Box 12 summarises the evidence discussed here on pharmacological treatment.

\section{Clinical practice}

The following fictitious vignette illustrates a combination of psychosocial and pharmacological interventions to manage anxiety in an elderly man with dementia and to support his wife, who is his primary carer.

B0X 12 Pharmacological management of anxiety in dementia

First-line drugs

Citalopram - has a safe profile and evidence of effectiveness in reducing anxiety and behavioural symptoms

Sertraline and fluoxetine - have a safe profile and evidence of effectiveness in reducing anxiety, but without improvement in behavioural symptoms

Second-line drugs

Clomipramine and imipramine - have an evidence base for the treatment of anxiety but are associated with cognitive decline

Benzodiazepines - could be considered for a short duration (effective but significant risk of adverse effects). Also consider in special circumstances (e.g. use of clonidine in dementia with Lewy bodies)

Acetylcholinesterase inhibitors - for anxiety-induced behavioural symptoms of dementia

Z-drugs (zopiclone, zolpidem, zaleplon) - where insomnia is secondary to anxiety

Third-line drugs

Venlafaxine, mirtazapine, pregabalin and buspirone have some evidence of effectiveness for anxiety in older people, but little or no evidence for their use in dementia Antipsychotics and anticonvulsants - their role is very restricted and they are associated with severe adverse effects

\section{Case vignette}

Mr Campbell is 78 years old and has a diagnosis of moderate vascular dementia. He scores 18 on the Mini-Mental State Examination (MMSE) and has become increasingly reliant on his wife for his activities of daily living. He has moments of lucidity, which Mrs Campbell values, but those moments are accompanied by an intense agitation that leaves her distraught. The agitation also affects Mr Campbell's sleep at night, as a consequence of which he is exhausted during the day. Mrs Campbell calls in his care coordinator, who establishes that there are days when Mr Campbell presents with excessive anxiety or worry which he finds difficult to control, together with restlessness, irritability, muscle tension, fears and respiratory distress. His general practitioner has checked him for infection and has assured Mrs Campbell that her husband is on adequate treatment for chronic obstructive pulmonary disease.

The care coordinator discusses the situation with the psychiatrist, who explores the possibility of anxiety comorbid with dementia and they undertake a behavioural analysis of $\mathrm{Mr}$ Campbell's agitation. One of the triggers for Mr Campbell's anxiety seems to be when he becomes aware that he has not been to the pub quiz in which he had participated regularly for more than 25 years. It is recommended that $\mathrm{Mr}$ Campbell try going to a day centre once a week, where group activities, including quizzes, take place. After 4 weeks, this seems to reduce his anxiety and Mrs Campbell is relieved, but episodes of anxiety contributing to poor sleep continue. Mr Campbell is offered a trial of mirtazapine $15 \mathrm{mg}$ at night and this helps with his sleep and reduces anxiety. During the 2 weeks of the trial, Mrs Campbell discusses sleep promotion and sleep hygiene with the care coordinator. This includes reducing light levels in the bedroom, removing mirrors, as these seem to provoke his anxiety, and giving him hot chocolate a bedtime, as he used to drink this to promote sleep when he was working irregular hours. Mrs Campbell begins a diary of her husband's anxiety, as carers in her local Alzheimer's Society support group have advised that this will be useful in providing personcentred care for her husband if, for example, he goes to a care home for respite care.

\section{Conclusions}

Anxiety is a common symptom leading to agitation in patients with dementia. There is limited research regarding how best to identify and manage anxiety in this group, although the flow chart in Fig. 1 outlines a basic procedure for use in practice. Future research should focus on epidemiological studies of the prevalence of anxiety in people with dementia, profiling the risk and aetiological factors and validating screening and diagnostic tools for this population. Studies will need to focus on behavioural and physiological symptoms and further trials of pharmacological and psychosocial interventions are needed. When patients present with behavioural and psychological symptoms of dementia, clinicians must be proactive in looking for and treating anxiety disorders. 


\begin{tabular}{|c|c|c|c|}
\hline \multicolumn{4}{|c|}{ Diagnosing anxiety } \\
\hline Corroborated history with behavioural analysis & $\begin{array}{r}\text { Phys } \\
\text { medication re }\end{array}$ & $\begin{array}{l}\text { history/ } \\
\text { cal examinat }\end{array}$ & $\begin{array}{l}\text { Rating scales such as RAID } \\
\text { Laboratory investigations }\end{array}$ \\
\hline \multicolumn{4}{|c|}{ Non-pharmacological interventions } \\
\hline Simple measures such as distraction & $\begin{array}{l}\text { Dementia-focused therapies } \\
\text { such as reminiscence }\end{array}$ & $\begin{array}{l}\text { CBT or problem-solving therapy, } \\
\text { if available }\end{array}$ & $\begin{array}{l}\text { Social interventions focused on } \\
\text { triggers such as loneliness }\end{array}$ \\
\hline \multicolumn{4}{|c|}{ Pharmacological interventions } \\
\hline $\begin{array}{c}\text { First line } \\
\text { Citalopram/sertraline/fluoxetine }\end{array}$ & $\begin{array}{r}\text { Tricycli } \\
\text { acetylch }\end{array}$ & $\begin{array}{l}\text { ne } \\
\text { azepines/ } \\
\text { e inhibitors }\end{array}$ & $\begin{array}{c}\text { Third line } \\
\text { Venlafaxine/mirtazapine/ } \\
\text { pregabalin/buspirone }\end{array}$ \\
\hline
\end{tabular}

Quick guide to management of anxiety in dementia. CBT, cognitive-behavioural therapy; RAID, Rating of Anxiety In Dementia scale.

\section{References}

Aarsland D, Cummings JL, Larsen JP (2001) Neuropsychiatric differences between Parkinson's disease with dementia and Alzheimer's disease. International Journal of Geriatric Psychiatry 16: 184-91.

Alexopoulos GS, Raue PJ, Dimitris N, et al (2011) Problem-solving therapy and supportive therapy in older adults with major depression and executive dysfunction: effect on disability. Archives of General Psychiatry 68: 33-41.

Alzheimer's Society (2011) Assistive technology - devices to help with everyday living. Alzheimer's Society (http://alzheimers.org.uk/site/ scripts/documents_info.php?documentlD=109).

American Psychiatric Association (1994) Diagnostic and Statistical Manual of Mental Disorders (4th edn) (DSM-IV). APA.

Baldwin D, Anderson I, Nutt D, et al (2005) Evidence-based guidelines for the pharmacological treatment of anxiety disorders: recommendations from the British Association for Psychopharmacology. Journal of Psychopharmacology 19: 567-96.

Ballard C, Neill D, O'Brien J, et al (2000) Anxiety, depression and psychosis in vascular dementia: prevalence and associations. Journal of Affective Disorders 59: 97-106.

Banerjee S, Smith SC, Lamping DL, et al (2006) Quality of life in dementia: more than just cognition. An analysis of associations with quality of life in dementia. Journal of Neurology, Neurosurgery and Psychiatry 77: 146-8.

Beekman AT, Bremmer MA, Deeg DJH, et al (1998) Anxiety disorders in later life: a report from the longitudinal aging study Amsterdam. International Journal of Geriatric Psychiatry 13: 717-26.

Bierman EJM, Comijs HC, Jonker C, et al (2009) The effect of anxiety and depression on decline of memory function in Alzheimer's disease. International Psychogeriatrics 21: 1142-7.

Bjelland I, Dahl AA, Haug TT, et al (2002) The validity of hospital anxiety and depression scale: an updated literature review. Journal of Psychosomatic Research 52: 69-77.

Bohm C, Robinson DS, Gammans RE, et al (1990) Buspirone therapy in anxious elderly patients: a controlled clinical trial. Journal of Clinical Psychopharmacology 10: 47S-51S

Chen JC, Borson S, Scanlan JM (2000) Stage-specific prevalence of behavioural symptoms in Alzheimer's disease in multi-ethnic community sample. American Journal of Geriatric Psychiatry 8: 123-33.

Cohen D, Luchins D, Eisdorfer C, et al (1990) Caring for relatives with Alzheimer's disease: the mental health risks to spouses, adult children and other family caregivers. Behavior, Health, and Aging 1: 171-82.

Cohen-Mansfield J, Billig N (1986) Agitated behaviour in the elderly: a conceptual review. Journal of American Geriatrics Society 34: 711-21.

Cummings JL, Mega M, Gray K, et al (1994) The neuropsychiatric inventory: comprehensive assessment of psychopathology in dementia. Neurology 44: 2308-14.
Derousene C, Rapin J, Lacomblez L, et al (2004) Memory complaints in 200 subjects meeting the diagnostic criteria for age-associated memory impairment: psychoaffective and cognitive correlates. Psychology and Neuropsychiatry 2: 67-74.

Evans S (2004) A survey of provision of psychological treatments to older adults in the NHS. Psychiatric Bulletin 28: 411-4.

Foy A, O'Connell D, Henry D, et al (1997) Benzodiazepine use as a cause of cognitive impairment in elderly hospital inpatients. JAMA 278: 27-31.

Gallacher J, Bayer A, Fish M, et al (2009) Does anxiety affect risk of dementia? Findings from Caerphilly prospective study. Psychosomatic Medicine 71: 659-66.

Gambi F, De Berardis D, Campanella D, et al (2005) Mirtazapine treatment of generalised anxiety disorder: a fixed dose, open-labelled study. Journal of Psychopharmacology 19: 483-7.

Gauthier S, Feldman H, Hecker J, et al (2002) Efficacy of donepezil on behavioural symptoms in patients with moderate to severe Alzheimer's disease. International Psychogeriatrics 14: 389-404.

Gorenstein E, Papp L (2007) Cognitive-behavioural therapy for anxiety in the elderly. Current Psychiatry Reports 9: 20-5.

Hancock GA, Woods B, Challis D, et al (2006) The needs of older people with dementia in residential care. International Journal of Geriatric Psychiatry 21: 43-9.

Katz IR, Reynolds CF, Alexopoulos GS, et al (2002) Venlafaxine ER as a treatment for generalised anxiety disorder in older adults: pooled analysis of five randomised placebo controlled trials. Journal of American Geriatric Society 50: 18-25.

Kinrys G, Wygant L (2005) Anticonvulsants in anxiety disorders. Current Psychiatry Reports 7: 258-67.

LaBarge $E$ (1993) A preliminary scale to measure the degree of worry among mildly demented Alzheimer disease patients. Physical \& Occupational Therapy in Geriatrics 11: 43-57.

Martinón-Torres G, Fioravanti M, Grimley Evans J (2004) Trazodone for agitation in dementia. Cochrane Database of Systematic Reviews issue 4: $C D 004990$

McIntyre A, Gendron A, McIntyre A (2006) Quetiapine adjunct to selective serotonin reuptake inhibitors or venlafaxine in patients with major depression, comorbid anxiety, and residual depressive symptoms: a randomized, placebo-controlled pilot study. Depression and Anxiety 24: 487-94.

Mendez MF, McMurtray A, Chen AK, et al (2006) Functional neuroimaging and presenting psychiatric features in frontotemporal dementia. Journal of Neurology, Neurosurgery and Psychiatry 77: 4-7.

Mintzer JE, Brawman-Mintzer 0 (1996) Agitation as a possible expression of generalised anxiety disorder in demented elderly patients: toward a treatment approach. Journal of Clinical Psychiatry 57 (suppl 7): 55-63.

\section{MCO answers \\ $1 \mathrm{a} \quad 2 \mathrm{~b} \quad 3 \mathrm{~d} \quad 4 \mathrm{a} \quad 5 \mathrm{~d}$}


Mohlman J, Gorenstein EE, Kleber M, et al (2003) Standard and enhanced cognitive behaviour therapy for late-life generalised anxiety disorder: two pilot investigations. American Journal of Geriatric Psychiatry 11: 24-32.

Montgomery S, Chatamra K, Pauer L, et al (2008) Efficacy and safety of pregabalin in elderly people with generalised anxiety disorder. British Journal of Psychiatry 193: 389-4.

Morinigo A, Blanco M, Labrador J, et al (2005) Risperidone for resistant anxiety in elderly patients. American Journal of Geriatric Psychiatry 13: $81-2$

Mynors-Wallis $L$ (2001) Problem-solving treatment in general psychiatric practice. Advances in Psychiatric Treatment 7: 417-25.

National Collaborating Centre for Mental Health (2006) Dementia: Supporting People with Dementia and their Carers in Health and Social Care (NICE Clinical Guideline 42). National Institute for Health and Clinical Excellence.

National Collaborating Centre for Mental Health (2009) Depression: The Treatment and Management of Depression in Adults (NICE Clinical Guideline 90). National Institute for Health and Clinical Excellence.

National Collaborating Centre for Mental Health, National Collaborating Centre for Primary Care (2011) Generalised Anxiety Disorder and Panic Disorder (with or without Agoraphobia) in Adults: Management in Primary, Secondary and Community Care (NICE Clinical Guideline 113). National Institute for Health and Clinical Excellence.

Neal M, Barton Wright P (2003) Validation therapy for dementia. Cochrane Database of Systematic Reviews issue 3: CD001394.

Neutal Cl, Hirdes JP, Maxwell CJ, et al (1996) New evidence on benzodiazepine use and falls: the time factor. Age and Ageing 24: 273-8.

Nordhus IH, Pallesen S (2003) Psychological treatment of late-life anxiety: an empirical review. Journal of Consulting and Clinical Psychology 71 643-51

Rabins PV, Blacker D, Rovner BW, et al (2007) American Psychiatric Association practice guideline for the treatment of patients with Alzheimer's disease and other dementias. Second edition. American Journal of Psychiatry 164 (suppl 12): 5-56.

Rozzini L, Chilovi BV, Peli M, et al (2009) Anxiety symptoms in mild cognitive impairment. International Journal of Geriatric Psychiatry 24 $300-5$

Scottish Intercollegiate Guidelines Network (2006) Management of Patients with Dementia: A National Clinical Guideline (no. 86). SIGN.

Seignourel PJ, Kunik ME, Snow L, et al (2008) Anxiety in dementia: a critical review. Clinical Psychological Review 28: 1071-82.
Shah A, Wadoo 0, Latoo J (2010) Psychological distress in carers of people with mental disorders. British Journal of Medical Practitioners 3: a327.

Shankar KK, Walker M, Frost D (1999) The development of a valid and reliable scale for rating anxiety in people with dementia (RAID). Aging \& Mental Health 3: 39-49.

Sink KC, Holden KF, Yaffe K (2005) Pharmacological treatment of neuropsychiatric symptoms of dementia: a review of the evidence. JAMA 293: 596-608.

Spector A, Davies S, Woods B, et al (2000) Reality orientation for dementia: a systematic review of the evidence of effectiveness for randomised controlled trials. Gerontologist 40: 206-12.

Spector A, Thorgrimsen L, Woods B, et al (2003) Efficacy of an evidence-based cognitive stimulation therapy programme for people with dementia: randomised controlled trial. British Journal of Psychiatry 183: $248-54$

Starkstein SE, Jorge R, Petracca G, et al (2007) The construct of generalised anxiety disorder in Alzheimer disease. American Journal of Geriatric Psychiatry 15: 42-9.

Thompson S, Herrmann N, Rapoport MJ, et al (2007) Efficacy and safety of antidepressants for treatment of depression in Alzheimer's disease: a meta analysis. Canadian Journal of Psychiatry 52: 248-55.

Tsuang MT (1978) Genetic counseling for psychiatric patients and their families. American Journal of Psychiatry 135: 1465-75.

Twelftree H, Oazi A (2006) Relationship between anxiety and agitation in dementia. Aging \& Mental Health 10: 362-7.

Wagner AK, Zhang F, Soumerai SB, et al (2004) Benzodiazepine use and hip fractures in the elderly: who is at greatest risk? [Article in German] Archives of Internal Medicine 164: 1567-72.

Wetterling T, Backhaus J, Junghanns K (2002) Addiction in the elderly an underestimated diagnosis in clinical practice? Nervenarzt 73: 861-6.

Wilson SJ, Nutt DJ, Alford C, et al (2010) British Association for Psychopharmacology consensus statement on evidence-based treatment of insomnia, parasomnias and circadian rhythm disorders. Journal of Psychopharmacology (BAP Consensus Guidelines Special Issue): 1-25.

Wing JK, Babor T, Brugha T, et al (1990) SCAN: Schedules for Clinical Assessment in Neuropsychiatry. Archives of General Psychiatry 47: 589-93.

Woods B, Spector AE, Jones CA, et al (2005) Reminiscence therapy for dementia. Cochrane Database of Systematic Reviews issue 2: CD001120.

\section{MCOs}

Select the single best option for each question stem

\section{Regarding the epidemiology of anxiety in elderly people:}

a generalised anxiety disorder is the most common late-life psychiatric disorder

b anxiety has been reported in at least $60 \%$ of patients with dementia

c the prevalence of anxiety in people with dementia is less than $10 \%$

$\mathrm{d}$ in people with dementia, the rate of anxiety is $10-15 \%$

e the prevalence of anxiety in patients presenting with dementia is lower than in community samples.

2 Risk factors for anxiety in dementia include: a age

b being Asian

c education d severe dementia

e gender.

3 Regarding anxiety in dementia:

a anxiety in mild cognitive impairment is associated with impaired verbal learning but not executive function

b clinically significant anxiety and neuroticism predict cognitive decline

c depression, but not anxiety, predicts a decline in learning in patients with early Alzheimer's dementia

d irritability can individually predict anxiety in dementia

e anxiety in dementia is associated with lack of insight into the disorder.

\section{Regarding diagnosis and management of} anxiety in dementia:

a antidepressants can cause anxiety

b the HADS is a useful diagnostic tool c the RAID correlates poorly with the DSM-IV diagnosis of generalised anxiety disorder

$\mathrm{d}$ validation therapy, but not reality orientation therapy, produces an improvement in mood

e the Worry Scale is a carer-report measure for anxiety in moderate dementia.

\section{Regarding treatment of anxiety and} depression in dementia:

a alprazolam is associated with fewer hangover effects than lorazepam

b buspirone, but not pregabalin, has trial evidence to support its use

c fluoxetine reduces anxiety and behavioural symptoms

$\mathrm{d}$ problem-solving therapy is beneficial in treating depression associated with executive dysfunction

e the success of CBT is independent of executive dysfunction. 